11. Les quartiers indurés ne donnent pas tous des streptocoques ou un nombre significatif de cellules.

12. Le lait d'un quartier normal ou exempt de sclérose ne contient pas plus de 150.000 streptocoques en longues chaînes ou cellules par centimètre cube.

13. Les recherches des proportions de chlorures, de lactose et de catalase existant dans le lait constituent des méthodes très utiles pour déceler les quartiers infectés. Toutefois, les méthodes adoptées pour procéder à ces essais ne sont pas encore telles qu'on puisse les utiliser comme de simples méthodes habituelles.

14. Des observations effectuées en examinant post-mortem 129 mamelles, qui avaient été préalablement examinées par palpation, indiquèrent qu'un examen physique ordinaire bien effectué est très efficace pour déceler la sclérose ou l'induration des tissus de la mamelle.

15. Des tests positifs avec le bol d'essai de traite et la réaction au bleu de bromothymol (alcalinité : $p \mathrm{H}$ 7,0) indiquèrent toujours un quartier anormal. D'un autre côté, des résultats négatifs n'indiquèrent pas toujours une mamelle normale.

\title{
ÉTUDE DE L'APPRÉCIATION DE LA QUALITÉ DES CASÉINES LACTIQUES
}

\author{
par \\ JEAN PIEN \\ Ingénieur chimiste I. C. R. \\ Docteur ès sciences \\ Directeur des laboratoires des "Fermiers Réunis"

RoBert MARTIN et MARC BERGIER
ngénieur chimiste I. C. R.
des laboratoires de la laiterie des "Fermiers Réunis "

(Suite.)

\section{§3. LA TECHNIQUE DU DOSAGE DES DIFFÉRENTES FORMES D'AZOTE SOLUBLE DANS LES CASÉINES INDUSTRIELLES}

I. Les formes d'azote à doser. - Nous avons indiqué, dans notre précédent article, une méthode permettant de déterminer globalement la teneur d'une caillebotte ou d'une caséine en azote. soluble dans l'eau.

Ce renseignement peut ne pas être suffişant. En effet : au cours de l'altération fermentaire d'une caillebotte, il peut se former à partir de la protéine pure des produits d'hydrolyse insolubles dans l'eau et ayant échappé, par conséquent, aux déterminations précé- 
dentes. On sait d'ailleurs qu'il peut exister, dans cette catégorie de matières azotées dégradées insolubles, des formes d'azote solubles dans les acides organiques. Enfin, si on a affaire à des fermentations putréfiantes assez poussées, il apparaît toujours de l'ammoniaque.

Donc, on peut penser que dans une caillebotte ou une caséine altérée, on peut rencontrer simultanément les différentes formes d'azote que voici :

Azote de la caséine pure inaltérée insoluble ;

Azote des formes dégradées insolubles dans l'eau, mais solubles dans un acide organique dilué ;

Azote soluble dans l'eau et les acides dilués ;

Azote ammoniacal.

Enfin, dans le cas très rare où la production d'ammoniaque aurait été suffisante, il peut y avoir réaction au sein de la masse entre l'azote de la caséine inaltérée et cette ammoniaque. On assiste alors à l'apparition de caséinate d'ammoniaque, forme nouvelle d'azote soluble dans l'eau, mais reprécipitable par les acides (forme tout à fait exceptionnelle. rencontrée seulement dans les caillebottes pourries).

Il est clair que l'interprétation de l'analyse sera très différente suivant que l'un ou l'autre de ces termes sera nul ou au contraire, représentera un pourcentage important de l'azote total.

La classification que nous venons de donner des différentes formes d'azote possibles dans une caséine altérée, n'a pas la prétention d'être parfaite et immuable. On pourrait imaginer d'autres subdivisions dans les formes d'azote entièrement soluble, par exemple. Il peut un jour devenir évident que la présence de tel ou tel groupement moléculaire, de telle ou telle fonction, de tel ou tel radical même, est absolument indésirable, pour tel ou tel usage industriel de la caséine. La question pourra et devra être reprise et fortement améliorée.

Pour le moment, nous nous eontenterons d'une classification un peu artificielle basée surtout sur des considérations d'ordre analytique. Mais cette classification tient déjà sérieusement compte des répercussions pratiques. En effet : il est extrêmement intéressant de savoir si une caséine donnée a atteint partiellement avant séchage le stade caséinate spontanément soluble dans l'eau; il peut être important de savoir si l'on se trouve en présence d'une caséine contenant des produits d'hydrolyse susceptibles de passer en solution dans l'eau, mais plus ou moins solubles également dans les acides dilués, ce qui correspondait à une caséine non entièrement reprécipitable de ses solutions alcalines, par exemple.

D'une manière générale, sans vouloir chercher une composition 
chimique détaillée des diverses formes d'azote de la caséine altérée, nous pensons que la connaissance de la répartition brute de l'azote entre les différentes formes énumérées ci-dessus peut déjà être d'un grand intérêt pratique pour l'appréciation de la qualité des produits.

$$
*^{*} *
$$

II. Les techniques de dosage. - Nous devons maintenant indiquer les techniques qui permettent de doser divers groupes de formes d'azote pour en déduire ensuite par le caleul, les éléments énumérés plus haut.

10 Dosage de 1'azote total. - La-méthode kjeldahl est trop connue pour qu'il soit nécessaire de la rappeler en détail. Notons toutefois que le catalyseur de choix est le sulfate de cuivre ; qu'il y a intérêt à ajouter pour l'attaque une certaine quantité de sulfate de potasse pour élever le point d'ébullition de l'acide et favoriser la destruction de la matière organique; on met donc à attaquer dans un ballon kjeldahl : 2 gr. de caséine, $1 \mathrm{gr}$. 5 de sulfate de cuivre cristallisé (il est absolument inutile de le déshydrater au préalable comme cela a été recommandé par certains auteurs), $10 \mathrm{gr}$. de sulfate de potasse exempt d'azote, et $25 \mathrm{~cm}^{3}$ d'acide sulfurique à 66 Bé.

Ilestindispensable de maintenir l'ébullition environ une demi-heure après la décomposition complète. La distillation se fait dans les conditions ordinaires (sans sulfure de sodium ou hypophosphite bien entendu parce qu'on n'a pas utilisé le mercure comme catalyseur). On recueille le distillat dans de l'acide normal.

$2^{\circ}$ Dosage de l'azote soluble total. - La technique a été donnée dans notre article précédent.

$3^{\circ}$ Dosage de la caséine pure inaltérée.- On pourrait penser que la différence entre les deux dosages précédents donnera l'azote de la caséine inaltérée. Il n'en est rien, car le dosage de l'azote soluble total laisse de côté, avec l'insoluble, les formes acidosolubles (mais non hydrosolubles) et risquerait de les faire compter comme caséine vraie. Il est done important d'avoir un chiffre de caséine débarrassée des formes acidosolubles.

Le traitement de la caséine initiale par un acide organique dilué, ne résoudrait pas la question, car on serait dans de mauvaises conditions pour extraire de petites quantités d'azote acidosoluble à partir d'un produit insoluble. En principe, il faut mettre la caséine en solution, la reprécipiter par un acide faible, qui, précisément, laissera en solution les formes qui y sont solubles.

Il y a là une difficulté technique à résoudre. Il s'agit en effet de mettre la caséine en solution sans l'altérer. Après un certain 
nombre d'essais préalables, voici le mode opératoire que nous avons arrêté :

$2 \mathrm{gr}$. de caséine finement broyée sont mis à macérer pendant une demi-heure dans $50 \mathrm{~cm}^{3}$ d'eau distillée froide. On ajoute ensuite peu à peu $5 \mathrm{~cm}^{3}$ de soude normale et on agite jusqu'à dissolution complète (en restant toujours à froid). Aussitôt la dissolution obtenue, on ajoute goutte à goutte et en agitant $2 \mathrm{~cm}^{3}$ d'acide acétique cristallisable. La caséine pure précipite. Il est essentiel d'ajouter l'acide lentement et en agitant vigoureusement, pour former un précipité aussi fin que possible, de manière à faciliter les lavages. Laisser reposer quelques minutes. Décanter sur filtre sans eendres. Le filtrat doit être très clair. Repasser au besoin les premières portions. Laver dans le bécher par décantations successives à l'eau faiblement acétique. Finalement, recueillir tout le précipité sur le filtre et laver encore trois fois à fond. Tout ces filtrats et eaux de lavage auront été recueillis directement dans un ballon kjeldahl.

Le précipité est lavé à l'alcool ou à l'éther (surtout si la caséine est grasse) (1). On y dose l'azote selon KJELdaHL. On obtient ainsi l'azote de la cáséine pure inaltérée.

Le filtrat est concentré en présence d'acide sulfurique et on y dose également l'azote par un kjeldahl.

Remarque. - On peut également recueillir l'insoluble sur double filtre taré et le peser. Si on y dose ensuite l'azote, on trouve que le rapport $\frac{\text { Caséine pure }}{\text { Azote }}$ est bien égal à 6,4 , ce qui, d'une part, donne une confirmation de ce coefficient assez souvent contesté, et, d'autre part, apporte la preuve que la caséine extraite par ce moyen est pure.

$4^{\circ}$ Dosage de 1'azote ammoniacal. - Cette détermination pourrait avoir lieu sur le filtrat du dosage précédent. Mais il est préférable de doser tout l'azote de ce filtrat et de doser, à part, l'azote ammoniacal sur une autre portion de caséine initiale.

Il est extrêmement difficile de trouver une méthode de dosage de l'ammoniaque qui ne soit pas en même temps une cause de production d'ammoniaque à partir du produit examimé. Dans le cas qui nous occupe, tous les procédés par distillation en présence d'alcalis sont à rejeter. La meilleure méthode nous paraît être celle de Froidevaux et VAN Den Berghe (2), que l'un de nous avait déjà

(1) Dans le cas de caséines très grasses, il peut être utile de faire une extraction par l'alcool chaud au Kumagawa comme nous le faisons dans l'analyse de la matière azotée des fromages.

(2) Chimie et Industrie 1920, p, 612. 
adaptée au cas du dosage de l'ammoniaque dans les cyanamides (1)

Le principe de la méthode est connu depuis longtemps : l'azote ammoniacal est déplaçable à froid par la soude caustique. Le procédé Froidevaux consiste à entraîner par un courant d'air l'ammoniaque libéré.

La réalisation du procédé se fait à l'aide de l'appareil ci-dessous :

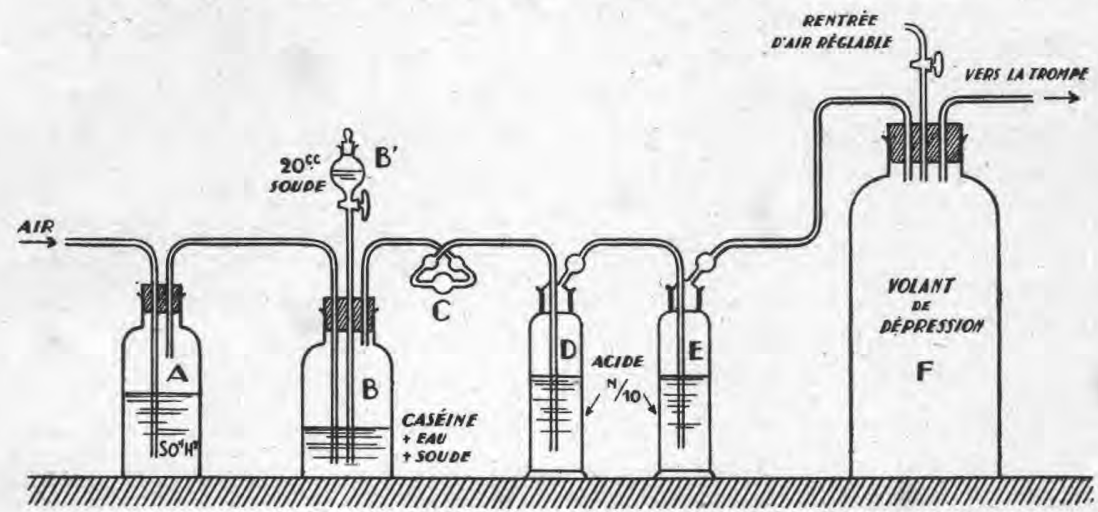

Appareil pour le dosage de l'azote ammoniacal, dans les caséines altérées.

A est un récipient contenant de l'acide sulfurique destiné à débarrasser l'air de la salle de l'ammoniaque qu'il pourrait contenir.

$\mathrm{B}$ reçoit le produit à examiner ainsi que la soude caustique primitivement contenue dans $\mathbf{B}^{\prime}$.

C est un dispositif de protection destiné à empêcher des entrainements mécaniques de soude.

$\mathrm{D}$ et $\mathrm{E}$ contiennent l'acide titré chargé de quelques gouttes d'indicateur coloré.

F est un volant de dépression destiné à régulariser la marche de l'entraînement.

Dans l'application que nous en faisons aux caséines, nous opérons ainsi :

5 gr. de caséine sont placés dans le récipient $\mathrm{B}$ avec $20 \mathrm{~cm}^{3}$ d'eau. B' reçoit $20 \mathrm{~cm}^{3}$ de soude caustique (robinet fermé). D et $\mathrm{E}$ reçoivent chacun $10 \mathrm{~cm}^{3}$ d'acide $\mathrm{N} / 10$.

On laisse d'abord gonfler la caséine pendant quelques minutes. Puis on met le vide et on règle le débit d'air de manière à avoir quelques bulles à la seconde. On ouvre alors doucement le robinet de l'ampoule $\mathbf{B}^{\prime}$. La soude dissout la caséine. On aura soin d'agiter souvent B pendant toute la durée de l'opération, qui dure environ 2 h. $1 / 2$.

(1) J. Pren. Etudes chimiques et agronomiques sur la cyanamide ealcique (thèse de Doctorat, Paris 1928). 
Le titrage en retour de l'excès d'acide des récipients $\mathrm{D}$ et $\mathrm{E}$ permet de calculer la quantité d'ammoniaque que contenait la caséine. (En principe $\mathrm{E}$ ne doit pas contenir d'ammoniaque ou seulement des traces.)

Remarque. - On pourrait penser que ce traitement de la caséine par de la soude concentrée à froid va altérer la caséine et peut-être produire de l'ammoniaque. En fait, avec des caséines très pures, préparées au laboratoire, on ne trouve que des traces d'ammoniaque par ce procédé (de l'ordre de $0,05 \%$ de la caséine). L'action de la soude à froid sur la caséine ne crée done pas d'ammoniaque.

$$
* *
$$

III. Calcul des différentes formes d'azote. - Chacune des techniques précédentes ne donne pas une seule forme d'azote. Il est done nécessaire de fixer les données et les inconnues du problème et d'écrire les équations nécessaires qui permettront de le résoudre.

$1^{\circ}$ Les inconnues. - Représentons chaque forme d'azote par une lettre, en laissant provisoirement de côté le cas exceptionnel des caséinates. Appelons (A) l'azote de la caséine pure inaltérée insoluble; (B) l'azote des formes dégradées insolubles dans l'eau mais solubles dans l'acide acétique dilué ; (C) l'azote entièrement soluble dans l'eau et les acides dilués ; (D) l'azote ammoniacal.

20 Les données. - Appelons Az l'azote total du produit initial donné par le kjeldahl ; S l'azote soluble dans l'eau (total) donné par la technique de l'article précédent; $\mathrm{Pa}$ l'azote de l'insoluble de la précipitation acétique; Fa l'azote du filtrat de la précipitation acétique ; Am l'azote ammoniacal.

$3^{\circ}$ Les équations. - Nous avons évidemment :

$$
\begin{aligned}
& \mathrm{Az}=(\mathrm{A})+(\mathrm{B})+(\mathrm{C})+(\mathrm{D}) \\
& \mathrm{S}=(\mathrm{C})+(\mathrm{D}) \\
& \mathrm{Pa}=(\mathrm{A}) \\
& \mathrm{Fa}=\mathrm{Az}-(\mathrm{A})=(\mathrm{B})+(\mathrm{C})+(\mathrm{D}) \\
& \mathrm{Am}=(\mathrm{D})
\end{aligned}
$$

Nous n'avons là en réalité que quatre équations, car (4) est implicitement contenue dans (1),

$4^{\circ}$ La solution. - Les équations précédentes permettent très facilement de calculer la valeur des inconnues en fonction des données.

Puisque $(\mathrm{A})=\mathrm{Pa}$, et $(\mathrm{D})=\mathrm{Am}$, nous tirons de (2) :

$$
\text { (C) }=\mathrm{S}-\mathrm{Am}
$$

Remplaçons (C) + (D) par sa valeur S dans l'équation (4) 
Il vient :

d'où :

$$
\mathrm{Fa}=(\mathrm{B})+\mathrm{S},
$$

$$
(\mathrm{B})=\mathrm{Fa}-\mathrm{S}
$$

Finalement les inconnues auront pour valeur :

$$
\begin{aligned}
& \text { (A) }=\mathrm{Pa} \\
& \text { (B) }=\mathrm{Fa}-\mathrm{S} \\
& \text { (C) }=\mathrm{S}-\mathrm{Am} \\
& (\mathrm{D})=\mathrm{Am}
\end{aligned}
$$

Il va de soi qu'on aurait pu, pour calculer (B), faire appel à l'équation (1) en faisant intervenir le terme Az. On aurait finalement :

$$
\text { (B) }=\mathrm{Az}-\mathrm{Pa}-\mathrm{S}
$$

Ce qui signifie que si on connaît $\mathrm{Az}$, on peut se dispenser de doser $\mathrm{Fa}$, ou réciproquement. Il est préférable de faire les-cinq déterminations, ce qui permet, dans une certaine mesure, des vérifications.

$5^{\circ}$ Cas des caséines très altérées (présence de caséinates). - Les calculs qui précèdent supposent évidemment qu'on ait toujours $\mathrm{Fa}>\mathrm{S}$.

Or, il nous est arrivé de rencontrer des caséines où cette inégalité n'était pas vérifiée et où le calcul de B était impossible.

Tel est précisément le cas des caséines provenant de caillebottes complètement putréfiées (cas très rare d'ailleurs) renfermant du caséinate d'ammoniaque. Que se passe-t-il alors?

$\mathrm{Si} \mathrm{Fa}$ est plus petit que S, c'est que la somme (Fa) des formes d'azote soluble dans l'eau et des formes acidosolubles, est inférieure à la somme des formes solubles dans l'eau (S). Cela peut signifier d'abord que les formes acidosolubles sont nulles en pareil cas et, en outre, qu'il doit exister des formes d'azote soluble dans l'eau (S) non solubles dans les acides, c'est-à-dire reprécipitables, et ne figurant pas en $\mathrm{Fa}$, qui se trouve ainsi devenir inférieur à $\mathrm{S}$. C'est bien le cas des caséinates solubles dans l'eau (S augmente) mais précipitables par l'acide acétique ( $\mathrm{Pa}$ augmente et $\mathrm{Fa}$ diminue). On s'explique bien ainsi qu'on puisse avoir $\mathrm{Fa}<\mathrm{S}$.

Le terme (B) n'aurait-il donc ici aucun sens ?

En réalité, on pourrait fort bien concevoir l'existence simultanée du terme $\mathrm{B}$ et du terme nouveau $\mathrm{E}$ (caséinates) pour vérifier l'inégalité $\mathrm{Fa}<\mathrm{S}$ rencontrée dans la pratique. Un calcul simple montrerait que la condition serait remplie tant que E serait supérieur à $\mathrm{B} / 2$. Mais si on accepte cette simultanéité, non démontrée d'ailleurs, de $\mathrm{B}$ et de $\mathrm{E}$, on introduit une cinquième inconnue dans un système à quatre équations et il n'y a plus de solution possible.

Si nous voulons tout de même nous faire une idée de la composi- 
tion approximative de ces caséines très altérées (et la chose présente beaucoup d'intérêt), il nous est indispensable, à défaut d'une équation nouvelle, d'admettre conventionnellement le terme B comme inexistant. Autrement dit, dans l'examen des caséines où $\mathrm{S}$ est supérieur à $\mathrm{Fa}$ (ce qui dénote une très grosse altération), nous supposerons toujours qu'il n'y a pas d'azote qui soit à la fois acétosoluble et insoluble dans l'eau. Cette convention, que rien n'interdit d'ailleurs d'une manière formelle ici, nous permettra des constatations analytiques fort intéressantes qui ont le mérite de concorder avec les constatations pratiques industrielles.

Jusqu'à plus ample informé, nous tenons donc cette convention pour acceptable $(B=0)$ et nous procéderons au calcul des caséines très altérées de la manière suivante :

Appelons E l'azote des caséinates, inconnue nouvelle. Cet azote est soluble dans l'eau et précipite par l'acide acétique ; les équations deviennent done :

$$
\begin{aligned}
& \mathrm{Az}=(\mathrm{A})+(\mathrm{C})+(\mathrm{D})+(\mathrm{E}) \\
& \mathrm{S}=(\mathrm{C})+(\mathrm{D})+(\mathrm{E}) \\
& \mathrm{Pa}=(\mathrm{A})+(\mathrm{E}) \\
& \mathrm{Fa}=\mathrm{Az}-(\mathrm{A})-(\mathrm{E})=(\mathrm{C})+(\mathrm{D}) \\
& \mathrm{Am}=(\mathrm{D})
\end{aligned}
$$

On en tire :

$$
\begin{aligned}
& (\mathrm{A})=\mathrm{Az}-\mathrm{S} \\
& (\mathrm{C})=\mathrm{Fa}-\mathrm{Am} \\
& (\mathrm{D})=\mathrm{Am} \\
& (\mathrm{E})=\mathrm{S}-\mathrm{Fa}
\end{aligned}
$$

Il va de soi qu'au lieu de faire intervenir $\mathrm{Az}$, on pourrait tenir compte de $\mathrm{Pa}$, et on aurait pour (A) la valeur $\mathrm{Pa},-$ (E) ou $\mathrm{Pa}+\mathrm{Fa}-\mathrm{S}$.

$$
*^{*} *
$$

\section{Application pratique.}

\section{Principe général.}

Etant donné une caséine, effectuer les cinq dosages prévus : $\mathrm{Az}$ (azote total), S (azote soluble total - voir article précédent), $\mathrm{Pa}$ et $\mathrm{Fa}$ (précipité et filtrat acétiques), Am (azote ammoniacal).

Si Fa est plus grand que $\mathrm{S}$, le terme $B$ existe et tous les éléments peuvent être calculés à l'aide de la première série d'équations.

Si Fa est plus petit que $\mathrm{S}$, on a affaire à une caséine très fortement altérée contenant des caséinates; il faut faire appel, pour le calcul, à la deuxième série d'équations, où ne figure pas le terme $\mathrm{B}$, mais où figure le terme $\mathrm{E}$ (azote des caséinates). 


\section{Exemples.}

Caséine $n^{0} 1$ : caséine très pure bien lavée non fermentée ;

Caséines n ${ }^{\circ} 2$ et 3 : caséines industrielles légèrement altérées ;

Caséines $n^{08} 4$ et 5 : caséines industrielles très altérées ;

Caséine $n^{\circ} 6$ : caséine fabriquée avec une caillebotte pourrie.

Donnons d'abord le résultat des dosages effectués :

\begin{tabular}{|c|c|c|c|c|c|c|}
\hline & 1 & 2 & 3 & 4 & 5 & 7 \\
\hline 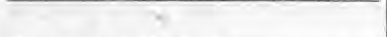 & & & & & & \\
\hline Azote total $(\mathrm{Az}) \ldots \ldots \ldots \ldots$ & 13,02 & 12,35 & 12,18 & 12,53 & 11,69 & 12,50 \\
\hline Azote soluble total (S) . . . . & 0,12 & 0,43 & 0,46 & 2,20 & 4,70 & 8,44 \\
\hline Insoluble de la précipitation & & & & & & \\
\hline acétique $(\mathrm{Pa}) \ldots \ldots \ldots$ & 12,67 & 11,62 & 11,55 & 9,73 & 6,09 & 7,14 \\
\hline Filtrat de la précipitation & & & & & & \\
\hline acétique $(\mathrm{Fa}) \ldots \ldots \ldots$ & 0,38 & 0,63 & 0,75 & 2,73 & 5,53 & 5,39 \\
\hline Azote ammoniacal $(\mathrm{Am}) \ldots$ & 0,05 & 0,17 & 0,27 & 0,18 & 0,29 & 0,35 \\
\hline
\end{tabular}

Dans les cinq premières caséines, on a $\mathrm{Fa}>\mathrm{S}$ et il faut appliquer à ces cinq caséines le premier groupe d'équations. Dans la sixième, on a $\mathrm{Fa}<\mathrm{S}$ et c'est le deuxième groupe d'équations qu'il faut appliquer. On obtient finalement :

\begin{tabular}{|c|c|c|c|c|c|c|}
\hline & 1 & 2 & 3 & 4 & 5 & 6 \\
\hline Azote de la caséine pure (A). & 12,67 & 11,62 & 11,55 & 9,73 & 6,09 & 4,06 \\
\hline Azote acétosoluble (B) & 0,26 & 0,20 & 0,29 & 0,53 & 0,83 & - \\
\hline $\begin{array}{l}\text { Azote hydrosoluble, non am- } \\
\text { moniacal (C) } \ldots \ldots \ldots \ldots\end{array}$ & 0,07 & 0,26 & 0,19 & 2,02 & 4,41 & 5,04 \\
\hline Azote ammoniacal (D) .... & 0,05 & 0,17 & 0,27 & 0,18 & 0,29 & 0,35 \\
\hline $\begin{array}{l}\text { Azote des caséinates }(\mathrm{E}) \ldots \\
\text { Somme des diverses formes }\end{array}$ & - & - & - & - & - & 3,05 \\
\hline d'azote $\ldots \ldots \ldots \ldots \ldots$ & 13,05 & 12,25 & 12,30 & 12,46 & 11,62 & 12,50 \\
\hline Azote total dosé directement & 13,02 & 12,35 & 12,18 & 12,53 & 11,69 & 12,50 \\
\hline
\end{tabular}

On peut enfin, pour rendre la conclusion plus saisissante, ramener tous les résultats à 100 d'azote total. Nous aurons ainsi le pourcentage d'altération sous ses diverses formes.

$3^{\circ}$ Interprétation des exemples. - Le tableau ei-après est extrêmement évocateur en ce qui concerne la répartition des diverses formes d'azote dans les caséines.

a) L'azote de la caséine pure décroît régulièrement au fur et à mesure de l'altération. Il était facile de le prévoir. Mais il est très intéressant de savoir que cette teneur peut considérablement 


\begin{tabular}{|c|c|c|c|c|c|c|}
\hline & 1 & 2 & 3 & 4 & 5 & 6 \\
\hline Azote total $\ldots \ldots \ldots \ldots$ & 100 & 100 & 100 & 100 & 100 & 100 \\
\hline Azote de la caséine pure (A). & 97,0 & 94,8 & 93,9 & 78,2 & 52,4 & 32,5 \\
\hline Azote acétosoluble (B) ... & 2,0 & 1,6 & 2,3 & 4,2 & 7,1 & - \\
\hline $\begin{array}{l}\text { Autres formes d'azote so- } \\
\text { luble (C) } \ldots \ldots \ldots \ldots \ldots\end{array}$ & traces & 2,1 & 1,5 & 16,2 & 37,9 & 40,3 \\
\hline Azote ammoniacal (D) .... & traces & 1,4 & 2,2 & 1,4 & 2,5 & 2,9 \\
\hline Azote des caséinates $(\mathrm{E}) \ldots$ & $\therefore$ & - & - & - & - & 24,4 \\
\hline
\end{tabular}

baisser sans que l'altération puisse être décelée par l'analyse brute et les caractères extérieurs.

b) Il semble que les caséines les plus pures contiennent, comme les caséines faiblement altërées, une petite quantité d'azote acétosoluble (mais insoluble dans l'eau), de l'ordre de $2 \%$ de l'azote total.

Cette quantité croît avec l'altération. Mais dans les caséines très fortement altérées (provenant de caillebottes pourries), il ne semble pas qu'on ait d'azote acétosoluble. En effet : la forte dose d'azote soluble (hydrosoluble) certain nous éloigne de l'hypothèse d'une forte dose d'acétosoluble que nos essais définissent comme nécessairement insoluble dans l'eau, ce qui rendrait plausible la convention admise plus haut $(B=0)$.

Sans doute, l'altération très prononcée de la caséine 6 commencet-elle précisément par l'attaque des formes acétosolubles, les rendant hydrosolubles. De nouvelles recherches seraient nécessaires pour éclaircir ce point.

c) L'azote ammoniacal ne prend pas, au cours de l'altération, l'importance qu'on serait tenté de lui attribuer. D'ailleurs, il faut bien admettre aussi que l'ammoniaque qui s'est formé dans les caillebottes altérées a été éliminé par le séchage de la caséine.

d) Les autres formes d'azote entièrement soluble dans l'eau (en dehors de l'ammoniaque et des caséinates éventuels) croissent régulièrement au cours de l'altération de la caséine initiale et e'est là peutêtre l'élément le plus intéressant au point de vue de l'utilisation industrielle.

e) Enfin, l'azote des caséinates, que l'on ne rencontre que dans les caséines très altérées, constitue un élément d'appréciation qui ne doit pas être confondu avec l'azote soluble vrai, non reprécipitable aux acides.

\section{Conclusions.}

1. L'analyse brute ordinaire des caséines (avec dosage de l'azote total comme seule forme d'azote) ne permet pas de déceler 
certaines altérations, même profondes, qui ne se révèlent pas toujours à l'aspect, mais qui se manifestent par des accidents de fabrication variés.

2. Les méthodes que nous avons exposées dans le présent article et dans le précédent, permettent de déterminer principalement le pourcentage de caséine pure vraie, qui est l'élément essentiel, et également les diverses formes d'azote soluble (hydrosoluble, acétosoluble, caséinates éventuels), qui constituent un élément d'appréciation complémentaire intéressant.

3. Il convient, bien entendu, que ces recherches soient reprises et complétées et surtout que l'observation industrielle soit menée parallèlement à l'étude chimique pour essayer d'établir un rapport entre la composition interne de la caséine et les accidents qu'elle peut provoquer dans les applications industrielles.

(A suivre.)

\title{
UNE MÉTHODE DE CONTROLE DE L'ALIMENTATION DES VACHES LAITIËRES (1)
}

\author{
par \\ M. ANDRÉ M. LEROY
}

Chef de travaux de Zootechnie à l'Institut National Agronomique.

Travail de la Station volante d'expérimentation zootechnique de l'Office régional du Nord.

(Fin.)

Une nouvelle question se pose maintenant. Cette différence entre les deux moyennes $\mathrm{C}$ et $\mathrm{T}$ est-elle significative ? Le calcul des probabilités nous apprend qu'il faut pour cela qu'elle soit au moins égale à deux fois, ou mieux, à trois fois son erreur probable.

Le calcul de cette erreur probable, d'après la formule $0,6745 \times \sqrt{\frac{\Sigma \mathrm{e}^{2}}{\mathrm{n}(\mathrm{n}-1)}}$, donne ici $\pm 0,35$. La différence ainsi mise en évidence mérite donc bien d'être prise en considération. Nous sommes en droit de l'attribuer à l'influence d'un régime alimentaire insuffisant, lors de la mise au régime d'hiver. Alerté à temps, le propriétaire de vaches laitières qui constate une pareille anomalie peut modifier en connaissance de cause les quantités de fourrages de ses rations et indiquer à ses vachers les bêtes qui devraient recevoir un supplément de nourriture : ce sont évidemment celles pour lesquelles il existe entre $\mathrm{C}$ et $\mathrm{T}$ une forte différence négative. Dans l'exemple

(1) Paru dans le Volume jubilaire du Professeur Ch. Porcher. 